\title{
Gambling in Asian Communities in Great Britain
}

\author{
David Forrest ${ }^{1 *}$, Heather Wardle ${ }^{2}$ \\ ${ }^{1}$ Maxwell Building, University of Salford, Salford, M5 4WT, United Kingdom; \\ ${ }^{2}$ Health and Wellbeing Department, National Centre for Social Research, 35 \\ Northampton Sq., London, ECIV 0AX, United Kingdom \\ * Corresponding author; email: d.k.forrest@salford.ac.uk
}

\begin{abstract}
This paper examines the prevalence of gambling and problem gambling among people of Indian, Pakistani, and Bangladeshi cultural backgrounds living in Great Britain. Information was gathered from the last two versions of a large-scale national adult prevalence survey and a similar survey of children aged 11 to 15 years, all conducted since 2007. Together, the surveys yielded sub-samples of 589 Asian adults and 482 Asian children from a combined total of more than 16,000 adults and nearly 9,000 children. In both adult and child Asian populations, the 7-day participation rate in gambling (22\% and $13 \%$, respectively) was found to be low relative to that in the white majority community, yet problem gambling prevalence, measured by the adult Diagnostic and Statistical Manual of Mental Disorders, 4th edition screen $(1.4 \%)$ and its juvenile equivalent $(2.9 \%)$, was significantly higher, including among women. It therefore follows that those Asians who do gamble are unusually prone to experiencing problems, which has implications for public health policies such as the provision of information and help in minority languages. The relevance of the findings seems likely to extend to other countries with significantly sized Asian communities.
\end{abstract}

Key words Asian Gambling $\cdot$ Problem Gambling

\section{Introduction}

A number of studies conducted in countries such as Australia and Canada have investigated gambling among Chinese communities; for a review, see Loo et al. (2008). Generally, these studies have drawn attention to the rates of gambling and problem gambling, which were found by the authors to be elevated relative to those in local majority populations.

In contrast to the Chinese population, minority overseas communities originating in other parts of Asia have been neglected in gambling studies. 
This paper therefore focuses on gambling behavior among the population whose cultural background is Indian, Pakistani, or Bangladeshi (henceforth referred by the shorter cultural designation, Asians).

Given the hostility to gambling in both Hindu and Muslim teaching, the activity might be expected to be widely socially disapproved of in Asian populations, but this does not mean that gambling cannot emerge within these groups as a significant source of personal and social problems. We have investigated whether gambling appears to be a source of problems because, if it does, health and social professionals need to be alert to the issue, and there may be a case for targeted interventions such as help lines and literature being provided in the relevant languages.

The setting for the study is Great Britain, which is appropriate for two reasons. First, Asians comprise a sufficiently large part of the population for it to be likely that large-scale general prevalence surveys will pick up sufficient observations of Asian people for meaningful statistical analysis to be undertaken (Asians, as defined here, accounted for $5.9 \%$ of the adult population in Great Britain according to the 2009 population estimates published by the Office of National Statistics [Office of National Statistics, 2011]). Second, three such gambling surveys have been conducted (on behalf of either the Gambling Commission or the National Lottery Commission) in the past four years, randomly sampling both adults (16 years and older) and children (aged 11-15 years) [Wardle et al., 2007 and 2011; Mori, 2009]. Together, these surveys offer researchers data on more than 25,000 respondents, including over 1,000 who self-identified as Asians (by our definition). The three surveys together paint a picture of gambling behavior in a setting where accessibility of gambling is very easy: the majority of the adult population is able to make regular use of the internet and lottery sales outlets, betting shops, slot machines and bingo halls are numerous throughout the country.

For children in the age-group that was surveyed, access is, however, restricted by legal limits. In general, the minimum age permitted for purchasing commercial gambling products is 18 years (16 years in the case of football pools and National Lottery draw games and scratch cards). However, slot machine play is allowed at any age so long as the prize is no more than GB£8 in tokens or GB£5 in cash (with stakes limited to 30 pence and 10 pence, respectively). Many arcades offer such machines and cater to a largely juvenile clientele. Of course, while stakes on each individual play are limited, this does not preclude larger amounts of money being spent during a session. Great Britain is unusual in permitting slot machine gambling at any age.

\section{Method}

\section{The adult surveys}

The information on adults was drawn from data generated by the British Gambling Prevalence Survey (BGPS). We pooled data from the last two editions, for which fieldwork was carried out by the National Centre for Social Research in the years 2006-2007 and 2009-2010, respectively. In each survey, the samples were based on random selection from a list of residential addresses in England, Scotland and Wales, and achieved close matches to the general population in terms of principal demographic characteristics (for technical details, see the official reports, Wardle 
et al., 2007, 2011). Interviewers visited each home to administer the questionnaire.

The questionnaire for each edition of the survey explored issues such as attitudes to gambling, participation in named modes of gambling, and intensity of involvement. Each edition also tested for problem gambling.

Pooling of the data from the two editions of the BGPS is extremely beneficial in terms of increasing the size of the Asian sub-sample, which is the focus of this analysis. While the findings of the two surveys indicated that there were some changes in overall gambling behavior between 2007 and 2010, for example a small increase in the proportion of past-year gamblers and variation in the relative popularity of some modes of gambling, these were not so great as to preclude combining the data sets, particularly since inspection of the data showed that broad associations between gambling behavior and various socio-economic and demographic variables were the same in each survey year. Pooling gave a sample of 589 Asians (as well as 15,241 whites, 382 blacks, and 359 who indicated another ethnicity such as Chinese or mixed background). Among these Asian adults, $48.8 \%$ had gambled in the preceding year and $21.8 \%$ in the preceding week. The proportion classified as problem gamblers according to the Diagnostic and Statistical Manual of Mental Disorders, 4th edition (DSM-IV; American Psychiatric Association, 2000) screen was $1.4 \%$.

\section{The child survey}

The data relating to children aged 11 to 15 years were generated from the British Survey of Children, the National Lottery and Gambling (2009), for which fieldwork was carried out by Mori during 2008-2009. This was a school-based survey, for which 8,958 children in 201 randomly selected schools in England, Scotland and Wales completed a questionnaire in class. In each school, two classes, one from school year 8 and one from school year 10, took part. Technical details of the sampling methods are included in the official report (Mori, 2009) and broad principles of the approach are outlined in Griffiths (2010). The survey asked children about gambling with their own money in the seven days preceding their completion of the questionnaire and about issues such as their use of alcohol, tobacco and drugs, and their parents' attitudes to gambling; a problem gambling screen, DSM-IV-Multiple Response Juvenile (DSM-IV-MR-J), was also included. Similar to the adult surveys, which excluded people who did not live at a residential address (for example those who were homeless or lived in institutions), the child survey did not include those who were outside the school system (for example, residents of juvenile detention centers). The final achieved sample size included 482 children who self-identified as Asians, 7,680 whites, 268 blacks, and 111 Chinese. As in the adult survey, a small number offered no answer to the ethnicity question. In total, $20.5 \%$ of children had spent their own money on one or more modes of gambling in the preceding seven days and $1.9 \%$ met the criteria of the screen for classification as a problem gambler.

\section{The problem gambling screens}

The BGPS applied two standard screens, DSM-IV and the Problem Gambling Severity Index (Wynne, 2003). The DSM-IV is more widely used internationally, and it is the results from this screen that will be reported here, mainly to facilitate 
comparison of evidence from the adult and child data. The children's survey included only a DSM-IV screen, albeit one adapted for use with minors, known as DSM-IV-MR-J (Fisher, 2000).

The adult and juvenile versions of the DSM-IV focus on similar dimensions of problem gambling. Each version contains questions about possible gambling dependency (preoccupation, needing to gamble with ever larger amounts of money, withdrawal symptoms, loss of control, gambling to escape, chasing losses) and questions about personal harm from gambling (resorting to lying, illegal acts, issues with relationships). However, the juvenile version adapts some of the questions to make them more relevant to children, for example reference to engaging in crime is replaced by asking whether they have ever used travel or dinner money or taken money that was not theirs to spend on gambling.

The questions in both the adult and child screens require respondents to choose from four ordered answers (e.g. never to often). Depending on the answer, one point may be scored. For example, in the child screen, only the strongest response, 'often', to the question "Have you found yourself thinking about gambling?", triggers one point to be scored. However, to the question "Has your gambling ever led to telling lies?", any of the three answers from 'once or twice' upwards scores one point. The screens can yield total scores between 0 and 10 ( 0 and 9 for children). Standard cutoffs classify a respondent as likely to be a problem gambler if he or she scores 3 or more (DSM-IV) or 4 or more (DSM-IV-MR-J). The reasons for these cut-off points are discussed by Wardle et al. (2011) and Fisher (2000).

Problem gambling measured by such criteria has consistently been found to be 2 to 4 times as high in adolescent populations as in adult populations in the same country (Valentine, 2008; Blinn-Pike et al., 2010). This has proved to be the case in the surveys described in this report; the pooled adult surveys estimated prevalence at $0.8 \%$, whereas the child survey recorded $1.9 \%$. However, while rooted in the same conceptual interpretation of what 'problem gambler' means, the adult and child versions of the screen are not identical, therefore, problem gambling rate estimates for adult and child populations are not strictly comparable. To an unknown extent, therefore, any measured difference between adult and child rates will reflect differences between the screens. However, this is not a major issue for this paper as the focus is on differences in gambling behavior between Asian adults and white adults and between Asian children and white children; each of these comparisons is between groups to which the same screen was administered. Whichever screen was used, it sought to measure some combination of dependent and self-harming behavior associated with gambling.

A caveat is that the screens used in the primary data sources were developed over time in the context of 'WEIRD' (western, educated, industrialized, rich, democratic) samples and may not reflect experience of gambling and problem gambling in a population of which many have come from a different cultural background. However, it is necessary to work with the available data, and comparisons between groups are most feasible if the same questionnaire has been used.

Where we make comparisons between groups, we report the statistical significance of any differences. All significance testing for adult data used the 
Statistical Package for the Social Sciences complex survey module (SPSS Inc., Chicago, IL, USA), which produces an adjusted Wald's F-test to model differences, taking into account the complex household sample design, weighting and sample clustering (Rao \& Scott, 1984). The children's data set does not have a complex, clustered, or household-based design so standard $t$-tests were appropriate in this situation.

\section{Results and Discussion}

\section{Gambling among Asian adults Attitudes to gambling}

Consistent with expectations, the BGPS data identified significantly less positive attitudes to gambling in the Asian than in the white population. Explicitly for the survey, researchers (Orford et al., 2009) had devised an attitude towards gambling scale (ATGS) designed to capture and quantify respondents' approval or disapproval of gambling. The ATGS was based on a series of statements on which respondents were invited to comment by indicating a response on a 5-point scale ranging from 'strongly agree' to 'strongly disagree'. Sometimes agreement with a particular statement signified sympathy with gambling and sometimes it revealed a negative attitude towards gambling. In all cases, the response most positive to gambling scored 5 points; the next response scored 4 points; and so on, until the most negative response scored just 1 point. The 2007 questionnaire included $14 \mathrm{such}$ statements, but this was reduced to eight core statements in 2010. The questions related to whether people should have the right to gamble whenever they want, whether there are too many opportunities to gamble, whether gambling should be discouraged, whether most people gamble sensibly, whether gambling is dangerous

Table 1. Mean attitude towards gambling scale (ATGS) scores among adults by sex and ethnic group (higher ATGS scores indicate more favorable attitudes towards gambling)

\begin{tabular}{lcccc}
\hline & \multicolumn{4}{c}{ Mean ATGS (\%) in each ethnic group } \\
\cline { 2 - 5 } & White & Asian & Black & Other \\
\hline All participants & & & $21.44^{*}$ & $20.74^{*}$ \\
\hline Men & 22.84 & $19.54^{*}$ & $19.59^{\dagger}$ & 19.99 \\
\hline Women & 21.06 & $17.70^{*}$ & $20.48^{*}$ & $20.33^{*}$ \\
\hline Total & 21.92 & $18.62^{*}$ & & \\
\hline Past-year gamblers & & & 22.68 & $21.97^{\dagger}$ \\
\hline Men & 23.44 & $20.83^{*}$ & 21.83 \\
\hline Women & 21.59 & $20.41^{\dagger}$ & 20.86 & 21.90 \\
\hline Total & 22.51 & $20.67^{*}$ & 21.91 & \\
\hline
\end{tabular}

${ }^{*}$ and ${ }^{\dagger}$ indicate significance at the $1 \%$ and $5 \%$ levels, respectively, where the null hypothesis is that there is no difference compared with the corresponding white group. 
for family life, whether it is good for society, whether it livens up life, and whether it would be better if gambling were banned. We pooled the results from 2007 and 2010 such that all the data for this report are based only on answers to these eight core questions. The scoring system allowed the ATGS score of any respondent to vary between 8 and 40, with higher scores signaling a more positive view of gambling.

The results indicate a general disinclination to hold and express extreme views for or against gambling, with a mean score of 21.71 across all respondents, a little lower than the mid-point of the scale, which is 24 . Table 1 shows the results by sex and ethnic group. Among men and women separately, the mean for Asians was of the order of magnitude of three-quarters of a standard deviation lower than that for whites. The difference between the Asian and white groups was significant ( $p<0.001$ and $p=0.003$ for men and women, respectively). Clearly there is less sympathy for gambling in the Asian community.

Attitudes towards gambling of individuals who had participated in gambling in the preceding year were more favorable, as shown in Table 1. However, the gap between Asian and white attitudes remained (the difference was significant at $p<$ 0.001 and $p=0.018$ for men and women, respectively).

It should be noted that the data presented here and throughout the discussion of the adult surveys are age-standardized. This is to permit comparisons between groups after adjusting for the effects of any differences in their age distributions. When different sub-groups are compared in respect of a variable on which age has an important influence, any differences in age distributions between these subgroups are likely to affect the observed differences in the proportions of interest. Age standardization, carried out using the direct standardization method, was undertaken separately within each sex group, expressing male data to the overall male population and female data to the overall female population. The standard population to which the age distribution of sub-groups was adjusted was given by the mid-year 2008 population estimates for England. Age standardization was not necessary in the case of the juvenile survey because differences in age distribution between sub-groups were negligible, given that the sample related to a narrow age range of $11-15$ years.

\section{Participation in gambling}

Reflecting attitudes, far lower participation in gambling was observed in the Asian than in the white sub-sample, although gambling was still a fairly widespread activity with more than $40 \%$ of Asian respondents reported having gambled in the year up to the date they completed the questionnaire and more than $20 \%$ reporting having gambled in the preceding seven days.

Table 2 presents 12-month and 7-day gambling participation rates by sex and ethnic group. Whites were more likely to have gambled than other groups, with the difference compared with Asians both very large and highly significant $(p<0.001$ for each sex). Participation within each ethnic group was higher for men, but the sex difference in behavior was relatively much greater for Asians (and also blacks). It may be stated with confidence that members of ethnic minorities, and Asians in particular, are much less likely to gamble than whites.

The survey asked about the modes of gambling in which respondents had 
Table 2. Adult gambling participation rates by sex and ethnic group

\begin{tabular}{|c|c|c|c|c|}
\hline \multirow[t]{2}{*}{ Gambling participation } & \multicolumn{4}{|c|}{ Gambling participation (proportion in each ethnic group) } \\
\hline & White & Asian & Black & Other \\
\hline \multicolumn{5}{|l|}{ Past-year gamblers } \\
\hline Men & 0.752 & $0.488^{*}$ & $0.623^{*}$ & $0.610^{*}$ \\
\hline Women & 0.708 & $0.331^{*}$ & $0.437^{*}$ & $0.529^{*}$ \\
\hline Total & 0.729 & $0.411^{*}$ & $0.526^{*}$ & $0.567^{*}$ \\
\hline \multicolumn{5}{|l|}{ Past-week gamblers } \\
\hline Men & 0.477 & $0.261^{*}$ & 0.440 & $0.340^{\dagger}$ \\
\hline Women & 0.398 & $0.173^{*}$ & $0.210^{*}$ & 0.368 \\
\hline Total & 0.437 & $0.218^{*}$ & $0.320^{*}$ & 0.355 \\
\hline \multicolumn{5}{|l|}{$\begin{array}{l}\text { Past-week participation in } \\
\text { individual modes }\end{array}$} \\
\hline National Lottery & 0.357 & $0.180^{*}$ & $0.269^{*}$ & 0.273 \\
\hline Scratch cards & 0.063 & 0.047 & 0.069 & 0.086 \\
\hline Other lottery & 0.040 & 0.036 & 0.023 & 0.025 \\
\hline Bingo & 0.027 & $0.003^{*}$ & 0.018 & 0.049 \\
\hline Football pools & 0.020 & $0.008^{\dagger}$ & 0.016 & 0.022 \\
\hline Slot machines & 0.032 & $0.013^{\dagger}$ & $0.015^{\dagger}$ & 0.023 \\
\hline FOBTs & 0.008 & 0.007 & 0.008 & 0.007 \\
\hline Table games in a casino & 0.004 & 0.008 & - & $0.015^{*}$ \\
\hline Online gambling & 0.010 & $0.003^{\dagger}$ & 0.002 & 0.009 \\
\hline Online betting & 0.009 & $0.001^{\dagger}$ & - & 0.009 \\
\hline Horse racing & 0.028 & $0.002^{+}$ & 0.024 & 0.010 \\
\hline Dog racing & 0.006 & - & 0.014 & 0.006 \\
\hline Other betting & 0.016 & $0.001^{*}$ & 0.027 & 0.010 \\
\hline Spread betting & 0.001 & - & $0.007^{\dagger}$ & 0.001 \\
\hline Betting exchange & 0.003 & 0.001 & - & 0.002 \\
\hline Private betting & 0.026 & $0.010^{\dagger}$ & 0.014 & 0.023 \\
\hline
\end{tabular}

${ }^{*}$ and ${ }^{\dagger}$ indicate significance at the $1 \%$ and $5 \%$ levels, respectively, where the null hypothesis is that there is no difference compared with the corresponding white group. 
participated in the preceding seven days, and the answers are summarized in Table 2. By far the most widespread mode of gambling in Britain is the National Lottery. Among whites, $38.0 \%$ of men and $33.6 \%$ of women had purchased a National Lottery ticket in the preceding week. Among Asians, the corresponding figures were $21.7 \%$ and $14.2 \%$ (significantly different from the figures for whites; $p<0.001$ for both sexes). Clearly, arithmetically, this difference drives the gap in participation in gambling as a whole. Nevertheless, the difference in propensity to gamble extends to most other modes. For example, slot machines attracted a little over $5.0 \%$ of white men but only $2.1 \%$ of Asian men, and betting on horse racing engaged in by just under $5 \%$ of white men, drew minimal interest among Asian men (much less than $1 \%$; $p$ values for differences were 0.056 and 0.006 , respectively). However, a curiosity appears in the data on use of Fixed Odds Betting Terminals, which are electronicroulette type gaming devices installed exclusively in bookmaker's offices. More Asian men reported having used them than had participated in betting, implying that some go to bookmaker shops only to play the machines. The proportion of men using such machines was not significantly different between the white $(1.5 \%)$ and Asian $(1.2 \%)$ sub-samples.

Whereas slot machines and betting have very low female participation in any ethnic group, bingo had been played by $3.7 \%$ of white women in the preceding week. By contrast, no Asian women interviewed in either survey year had played bingo, implying exclusion or self-exclusion from an activity outside the home that tends to be popular in poorer areas (blacks had only slightly lower participation and members of 'other' groups had higher participation than whites).

Next to the National Lottery draw, scratch cards represent the second most popular mode of gambling in the whole population. Here, the difference between ethnic groups was much less sharply defined. Among whites, $6.1 \%$ of men and $6.5 \%$ of women had played in the preceding seven days compared with $5.6 \%$ of Asian men and $3.8 \%$ of Asian women. The difference was not statistically significant for men or for women (among black men, purchase of scratch cards was high, with $10.0 \%$ reported participation). For private betting and lotteries other than the National Lottery, participation rates were also similar between Asian and white women.

\section{Prevalence of problem gambling}

The evidence shows that engagement in most modes of gambling is much less widespread in the Asian than in the majority white community, although this does not necessarily result in a lower prevalence of problem gambling.

Table 3 illustrates that problem gambling is an issue in the Asian population to the extent that problem gambling is just as prevalent as for whites. Focusing on the proportions of respondents scoring 3 or more on the DSM-IV screen, problem gambling prevalence was higher for all three minority groups than for whites, although differences were generally not statistically significant; this result is unsurprising given that problem gambling is relatively rare, affecting $1.23 \%$ of the whole male sample and $0.26 \%$ of the whole female sample, and that estimates are therefore made with imprecision. An exception is found among Asian women in that $1.34 \%$ scored 3 or more on DSM-IV compared with only $0.20 \%$ of white 
Table 3. Adult Diagnostic and Statistical Manual of Mental Disorders, $4^{\text {th }}$ edition (DSM-IV) scores by sex and ethnic group

\begin{tabular}{ccccc}
\hline \multirow{2}{*}{ DSM-IV score } & \multicolumn{4}{c}{ Proportion in each ethnic group } \\
\cline { 2 - 5 } & White & Asian & Black & Other \\
\hline Men & & & & \\
\hline 0 & 0.932 & 0.928 & $0.862^{\dagger}$ & 0.905 \\
\hline $1-2$ & 0.056 & 0.057 & $0.119^{\dagger}$ & 0.071 \\
\hline $3+$ & 0.012 & 0.014 & 0.018 & 0.024 \\
\hline Women & & & & \\
\hline 0 & 0.966 & 0.962 & 0.943 & 0.974 \\
\hline $1-2$ & 0.032 & 0.025 & 0.053 & 0.023 \\
\hline $3+$ & 0.002 & $0.013^{*}$ & 0.005 & 0.003 \\
\hline
\end{tabular}

${ }^{*}$ and ${ }^{\dagger}$ indicate significance at the $1 \%$ and $5 \%$ levels, respectively, where the null hypothesis is that there is no difference compared with the corresponding white group.

women. Of course, these proportions are based on identifying only small absolute numbers of problem gamblers, but the difference between Asian and white women is significant at $p=0.001$. That problems were signaled among Asian women is more remarkable given that the seven-day participation-rate is almost exactly half that of white women.

For both men and women, problem gambling is at least as common in the Asian community as in the white population. Evidently, low engagement rates in gambling do not grant immunity from problem gambling. Low-participation but high-problem gambling prevalence together imply that the risks from gambling conditional on taking part in gambling must be sharply elevated among Asians compared with whites, and this has been shown to be the case. We considered DSM-IV scores only among those in the sample who reported gambling activity in the preceding year. For men, the prevalence was $1.58 \%$ for whites and $3.36 \%$ for Asians (the difference was significant at $p=0.064$ ). For women, the prevalence was $0.29 \%$ for whites and $5.69 \%$ for women (the difference was significant at $p<0.001$ ). Similarly elevated rates were observed for Asians in terms of the numbers who scored either 1 or 2 on the DSM-IV scale. Among other ethnic groups, the same phenomena were observed for blacks, but only for men.

\section{Gambling among Asian children Attitudes of parents}

It is an important strength of this review that we are able to analyze behavior among adolescents as well as among adults. Blinn-Pike et al. (2010), in a major review of the literature on adolescent gambling, noted the lack of attention to racial and ethnic 
differences in gambling and problem gambling patterns in this age-group as one of its principal failings.

Comparisons will be made with the adult findings, but it should be noted that ethnic groups are defined slightly differently in the data from the two surveys. In the child survey, those who self-identified as Chinese are treated as a separate group, rather than being subsumed within 'others'. Further, the Asian group now includes all Asians other than Chinese. However, while this implies the inclusion of, for example, Vietnamese children, we know from Census data that the grouping is likely to be very heavily dominated by people from an Indian, Pakistani, and Bangladeshi cultural background (Office of National Statistics, 2011). We will therefore interpret findings for 'Asians' as illuminating behavior in those particular ethnic groups.

Where children were asked about their parents' attitudes to and participation in gambling, their combined responses displayed similar patterns as might have been anticipated from the adult surveys. The questionnaire asked them (separately) whether their parents would think it 'okay' for a child of their age to play the lottery or fruit machines (responses allowed for one parent to think differently from the other). If the children claimed that their parents thought it was acceptable in either or both cases or else had ambiguous views, we coded them as coming from gambling-tolerant homes. Among whites, 30.2\% of children were in this category, a much higher proportion than similar categories for drinking and smoking (on which similar questions had been asked and where parents' awareness of potential harm may be greater). However, in other ethnic groups, fewer parents were relaxed about gambling and only $18.7 \%$ of Asian children came from a gambling-tolerant home (the difference from whites was significant at $p<0.001$ ).

Similarly, when children were asked whether their parents played the lottery or bet with bookmakers, aggregated answers were consistent with patterns of participation evident in the adult data. Among whites, $61.1 \%$ had parents who bought lottery tickets (no time frame was specified) and $11.3 \%$ had parents who bet with bookmakers. For Asians, the corresponding figures were 37.8\% and 3.9\% (the differences from whites for each behavior were significant at $p<0.001$ ).

Just as one would expect, therefore, Asian children are less likely to come from homes where gambling is approved of or practiced than are white children. As both parental approval and parental gambling are recognized as prime risk factors for adolescent gambling and problem gambling (Griffiths, 2002), it might then be anticipated that prevalence of both would be lower among Asians. This proved to be the case for gambling but not for problem gambling.

\section{Participation in gambling}

The researchers who designed the questionnaire for the child survey (Fisher, 2000) chose to ask only about participation in the preceding seven days because they thought this would be highly correlated with regular gambling (they also took the view that a past-year participation question would have stretched children's ability to recall and date past behavior). Past-week participation was reported by $20.5 \%$ of the sample. Table 4 shows the participation rates by sex and ethnic group. Generally, it is difficult to identify statistically significant differences between 
whites and minority groups because of low sub-sample sizes for black and Chinese people, a problem that extends to Asians if they are divided by sex (hence we do not tabulate by sex for participation in individual modes). However, it is clear that a higher proportion of white children gamble than Asian children (the difference is significant at $p<0.001$ ). The gap is more evident among boys than among girls.

Generally, the more widespread popularity of gambling among white children, at least compared with their Asian counterparts, was mirrored in participation rates for individual modes. However, an exception was the purchase of tickets for the National Lottery, which was reported by only $1.4 \%$ of white children (and $0.7 \%$ of black children), while participation was 2.7\% for Asians (and 6.3\% for Chinese children). Asian and Chinese boys were principally responsible for this finding, with participation rates reaching $4.3 \%$ and $7.3 \%$, respectively. The differences from the white participation rates for both sexes together were significant at $p=0.02$ (Asians) and $p<0.001$ (Chinese). Among the Chinese group, scratch cards, also a National Lottery product, attracted much higher participation than among whites $(p<0.01)$ and there was an indication that this was also the situation for black children $(p=$ 0.06). Overall, there might be cause for concern over whether age restrictions on National Lottery products are enforced effectively for ethnic minority children. It

Table 4. Children's past-week gambling participation rates, by sex and ethnic group

\begin{tabular}{lllll}
\hline \multirow{2}{*}{ Gambling participation } & \multicolumn{3}{c}{ Proportion in each ethnic group } \\
\cline { 2 - 5 } & White & Asian & Black & Other \\
\hline Any gambling & & & & \\
\hline Boys & 0.292 & $0.157^{*}$ & 0.278 & 0.200 \\
\hline Girls & 0.125 & 0.108 & 0.145 & 0.107 \\
\hline Total & 0.208 & 0.133 & 0.220 & 0.153 \\
\hline Past-week participation in individual modes & & & & \\
\hline National Lottery & 0.014 & $0.027^{\dagger}$ & 0.007 & $0.063^{*}$ \\
\hline National Lottery scratch cards & 0.006 & $0.008^{*}$ & 0.015 & 0.027 \\
\hline Slot machines & 0.079 & $0.031^{*}$ & 0.075 & 0.054 \\
\hline Bingo & 0.010 & 0.010 & 0.011 & 0.018 \\
\hline Bookmaker betting & 0.018 & 0.012 & 0.022 & 0.009 \\
\hline Betting with friends & 0.075 & 0.052 & 0.078 & 0.063 \\
\hline Playing cards & 0.069 & 0.041 & 0.056 & 0.072 \\
\hline Gambling websites & 0.015 & 0.012 & 0.015 & 0.009 \\
\hline
\end{tabular}

${ }^{*}$ and ${ }^{\dagger}$ indicate significance at the $1 \%$ and $5 \%$ levels, respectively, where the null hypothesis is that there is no difference compared with the corresponding white group. 
might be relevant to note that most children who evaded the restrictions reported having bought tickets at a convenience store (Mori, 2009), presumably because supermarkets more consistently check age, and such stores are themselves very commonly operated by members of ethnic minority groups.

Apart from the anomaly of the lottery products, similar patterns were observed across all four groups in apparent popularity of different modes of gambling. Betting with friends and slot machines were the modes of choice. Both of these modes are normally legal in Great Britain. Of course, in measuring children's choices among modes, we are not observing true demand because legal constraints push their participation towards those where such obstacles are absent or laxly enforced. Indeed, Valentine (2008) suggests that children will substitute gambling among themselves in jurisdictions where regulatory impediments to taking part in commercial gambling are made most strict.

\section{Prevalence of problem gambling}

Parental attitudes and behavior appear, then, to restrain Asian children in the 1115 years' age-group to the extent that there are only about half the proportion of gamblers as in the white group. However, as noted for adults, low prevalence of gambling does not necessarily imply low prevalence of problem gambling.

Table 5 shows the proportions of problem gamblers according to the criterion of a score of 4 or more on the DSM-IV-MR-J screen. The proportion is lowest for whites $(1.8 \%)$ and highest for Asians $(2.9 \%)$ for a $p$ value of 0.08 . We can conclude that problem gambling is at least as high among Asians notwithstanding that Asians are significantly less likely to be gamblers. This mirrors the paradoxical result from analysis of the adult data and the resolution of the paradox is the same - Asian children are very much more likely to be experiencing problems if they are gamblers relative to white children who are gamblers.

We also calculated the problem gambling prevalence rate among past-week child gamblers, which was 7.0\% for whites, $6.8 \%$ for blacks, $18.7 \%$ for Asians, and $17.6 \%$ for Chinese. These rankings between ethnic groups are exactly the reverse of rankings of participation rates.

Asian children are therefore very much more likely than white children to have problems if they are gambling $(p<0.001)$. For Chinese children, any gambling

Table 5. Child problem gambling rates (Diagnostic and Statistical Manual of Mental Disorders, 4th edition [DSM-IV]-MR-J) by ethnic group

\begin{tabular}{lllll}
\hline \multirow{2}{*}{ DSM-IV-MR-J score } & \multicolumn{4}{c}{ Proportion in each ethnic group } \\
\cline { 2 - 5 } & White & Asian & Black & Other \\
\hline Boys & 0.028 & 0.043 & 0.040 & 0.000 \\
\hline Girls & 0.008 & 0.013 & 0.000 & $0.054^{*}$ \\
\hline Total & 0.018 & 0.029 & 0.022 & 0.027 \\
\hline
\end{tabular}

* indicates significance at the $1 \%$ level where the null hypothesis is that there is no difference compared with the corresponding white group. 
also appears to have a high chance of becoming problematic, although the subsample for this group is small and only three problem gamblers, all girls, were identified.

\section{Asian problem gamblers}

Large-scale prevalence surveys are invaluable for revealing relative prevalence rates across ethnic groups because, while a sample of problem gamblers in treatment is easier and cheaper to access, it is highly unlikely to be representative of all problem gamblers because it is self-selected, and members of minority communities may find it hardest to seek help and therefore be least likely to present with a problem. On the other hand, it is a disadvantage of the population-survey approach that absolute numbers of problem gamblers identified and available for further study are low. This is true for both adult and child surveys. Nevertheless we looked at the full set of responses of the 14 Asian child gamblers who were observed in our data to check for any attributes that were common across the group.

The modes in which the 14 child gamblers participated were not obviously skewed in any one direction. Five participants played slot machines, but this is anyway the most popular mode among the whole sample. Only one participant reported having gambled using the internet, so e-gaming does not appear to be a critical threat in terms of child problem gambling. Five participants indicated that they had drunk alcohol in the preceding seven days (which was rare in the whole Asian sub-sample, in which less than 9\% had drunk alcohol), but this is unexceptional given the high comorbidity in all problem gambling populations. There was no evidence that these 14 children were disadvantaged by deprived backgrounds; only three children attended schools where the proportion of pupils entitled to free school meals (on account of their parents being on welfare) was above the national average of $10.6 \%$.

Perhaps the sole characteristic that stood out was the high level of income available to these children. The respondents to the survey had been asked to indicate how much income they received each week and 11 of the 14 children reported a figure that placed them in the top quintile of the whole Asian child subsample. This is consistent with findings from analysis of the full child data set by Forrest and McHale (2011). In regression models, which controlled for a rich set of individual, home and school characteristics, these researchers established that level of pocket money was a powerful risk factor for both participation in gambling and for problem gambling conditional on participation.

\section{Conclusions}

The results revealed in the adult and child analyses are strikingly similar. Asians are relatively unlikely to participate in gambling, but are relatively likely to exhibit signs of problem gambling if they do participate. Indeed, such is the elevated risk of problem gambling among Asians who gamble that the incidence of problem gambling is higher in the general Asian than in the general white population.

The greater prevalence of problem gambling among Asians does not appear to be the result merely of composition effects. In the report on the last BGPS (Wardle et al., 2011), being Asian was shown to be a predictor of problem gambling even 
after controlling for income, socio-economic status, and level of deprivation of area of residence. Similarly, the report on the child survey (Mori, 2009) showed results from a regression model to account for DSM-IV-MR-J score and being Asian was a powerful positive predictor in the presence of a large number of variables representing home and school characteristics. Thus, it is not the case that elevated prevalence of problem gambling among Asians can readily be attributed to Asians living disproportionately often in deprived homes or areas where problem gambling risks may be relatively high. Rather 'Asian' appears to be an independent risk factor for both adults and children.

Why Asians in Great Britain are so prone to problem gambling if they do gamble remains a matter for speculation or for further research. Given the unambiguous findings from the ATGS questions in the BGPS, we know that gambling is less approved of among Great Britain's Asian population than among the white population. Those who gamble must therefore overcome the cost of social disapproval if they decide to gamble; and those willing to pay that cost may be those who are most drawn to gambling by unobserved personal characteristics, such as love of risk, which also predisposes them to problem gambling. Further, a negative attitude to gambling among Asians is not conducive to gambling emerging as a social pastime in that community and those who do gamble are therefore instead disproportionately likely to be taking part for relieving tension, motive associated with high problem gambling risk. The 2010 BGPS asked regular gamblers their reasons for gambling and Asians were far more likely than whites to endorse items that were categorized in the report as 'coping' motives for gambling (Wardle et al., 2011, pp.120-125h).

Another issue is that gambling in the white population is well entrenched and relatively little stigmatized, permitting some learning of moderation from the example of others and informal support for those whose behavior approaches problematic. However, problem gambling is an issue in the Asian population, yet the community as a whole may have little knowledge or experience of gambling (shown by the low participation rates). This reinforces the argument that provision of education, information, and treatment services may be all the more urgently required and appropriate. Globalization has spawned Asian communities in many countries outside of Europe and this evidence from Great Britain suggests that they should not be neglected by those responsible for provision of services designed to mitigate the harm that gambling can inflict on individuals, their families, and their communities.

\section{References}

American Psychiatric Association. (2000). Diagnostic and statistical manual of mental disorders, $4^{\text {th }}$ edition, text revised. Washington, DC: American Psychiatric Association.

Blinn-Pike, L., Worthy, S. L., \& Jonkman, J. L. (2010). Adolescent gambling: a review of an emerging field of research. Journal of Adolescent Health, 47, 223-236.

Fisher, S. (2000). Developing the DSM-IV criteria to identify adolescent gambling in nonclinical populations. Journal of Gambling Studies, 16, 253-273.

Forrest, D., \& McHale, I. G. (2011). Problem gambling among young adolescents in Great Britain, discussion paper, University of Salford.

Griffiths, M. D. (2002). Gambling and gaming addictions in adolescence. Leicester: The British 
Psychological Society and Blackwell Publishers Ltd.

Griffiths, M. D. (2010). Asian national adolescent gambling surveys: methodological issues, protocol and advice. Asian Journal of Gambling Issues and Public Health, 1, 4-18.

Loo, J. M. Y., Raylu, N., \& Oei, T. P. S. (2008). Gambling among the Chinese: A comprehensive review. Clinical Psychology Review, 21, 157-178.

Mori, I. (2009). British survey of children, the National Lottery and Gambling: Report of a quantitative survey. London: The National Lottery Commission.

Office of National Statistics. (2011). Population estimates by ethnic group 2002-2009. Retrieved from http: / / www.statistics.gov.uk/pdfdir/ eth0511.pdf

Orford, J., Griffiths, M., Wardle, H., Sproston, K., \& Erens, B. (2009). Negative public attitudes towards gambling: findings from the 2007 British Gambling Prevalence Survey using a new attitude scale. International Gambling Studies, 9, 39-54.

Rao, J. N. K., \& Scott, A. J. (1984). On chi-squared test for multiway contingency tables with cell proportions estimated from survey data. The Annals of Statistics, 12, 46-60.

Valentine, G. (2008). Literature review of children and young people's gambling. Birmingham: The Gambling Commission.

Wardle, H., Sproston, K., Orford, J., Erens, B., Griffiths, M. D., Constantine, R., \& Pigott, S. (2007). The British gambling prevalence survey 2007. London: The Stationery Office.

Wardle, H., Moody, A., Spence, S., Orford, J., Volberg, R., Jotangia, ... Dobbie, F. (2011). British gambling prevalence survey 2010. London: The Stationery Office.

Wynne, H. J. (2003). Introducing the Canadian Problem Gambling Index. Edmonton, AB, Canada: Wynne Resources. 